\title{
Multivariate Time-Varying $G-H$ Copula GARCH Model and Its Application in the Financial Market Risk Measurement
}

\author{
Qi-an Chen, ${ }^{1}$ Dan Wang, ${ }^{1}$ and Mingyong Pan $^{2}$ \\ ${ }^{1}$ School of Economics and Business Administration, Chongqing University, Chongqing 400030, China \\ ${ }^{2}$ School of Mathematics and Statistics, Chongqing University, Chongqing 400030, China \\ Correspondence should be addressed to Qi-an Chen; chenqi_an33@163.com and Mingyong Pan; panmingyong@aliyun.com
}

Received 23 March 2015; Accepted 11 May 2015

Academic Editor: Ruihua Liu

Copyright (c) 2015 Qi-an Chen et al. This is an open access article distributed under the Creative Commons Attribution License, which permits unrestricted use, distribution, and reproduction in any medium, provided the original work is properly cited.

\begin{abstract}
Taking full advantage of the strengths of $G-H$ distribution, Copula function, and GARCH model in depicting the return distribution of financial asset, we construct the multivariate time-varying $G-H$ Copula GARCH model which can comprehensively describe "asymmetric, leptokurtic, and heavy-tail" characteristics, the time-varying volatility characteristics, and the extreme-tail dependence characteristics of financial asset return. Based on the conditional maximum likelihood estimator and IFM method, we propose the estimation algorithm of model parameters. Using the quantile function and simulation method, we propose the calculation algorithm of VaR on the basis of this model. To apply this model on studying a real financial market risk, we select the SSCI (China), HSI (Hong Kong, China), TAIEX (Taiwan, China), and SP500 (USA) from January 3, 2000, to June 18, 2010, as the samples to estimate the model parameters and to measure the VaRs of various index risk portfolios under different confidence levels empirically. The results of the application example are in line with the actual situation and the risk diversification theory of portfolio. To a certain extent, these results also justify the feasibility and effectiveness of the multivariate time-varying $G$ - $H$ Copula GARCH model in depicting the return distribution of financial assets.
\end{abstract}

\section{Introduction}

Financial market risk has always been one of the hottest topics in the field of financial investment, and many financial researchers put forward many different financial market risk measurement methods. Among them, Value-at-Risk (VaR) management technology is an assessment and measurement method of financial risk that has risen in recent years, playing an increasingly important role in the risk management and investment decision. It has been widely adopted by the major banks, nonbank financial intermediaries, corporations, and financial regulators in the world and has become the standard of risk measurement and risk management in financial industry. Accurate calculation of $\mathrm{VaR}$ is one of the keys to estimate the probability distribution of future return on financial assets. Usually, it is assumed that financial asset returns are independent of each other and obey the normal distribution in the calculation of $\mathrm{VaR}$, but the movement of financial asset return in the financial market is extremely complex. The return of all kinds of financial assets usually does not satisfy the normal distribution hypothesis. However, it often shows "asymmetric, leptokurtic, and heavy-tail" characteristics [1-4]. At the same time, various financial asset returns do not satisfy the multivariate normal distribution hypothesis and present the extreme-tail dependence. On this occasion, a large error would be made by using normal distribution to fit financial asset return, and the estimation of the VaR may be overestimated or underestimated. To solve this problem, many scholars have proposed a lot of leptokurtic and heavy-tail distributions in recent years, such as the logistic distribution, Student's $t$-distribution, and the $G-H$ distribution. The logistic distribution and Student's $t$ distribution can comprehensively describe the leptokurtic characteristics of financial asset return series, but they could not make a good explanation for the heavy-tail characteristics of financial asset return series [5]. The $G-H$ distribution can comprehensively describe the asymmetric, leptokurtic, and heavy-tail characteristics of financial asset return series and it has a good fitting of the univariate unconditional return distribution of some financial assets; however, it could not 
reflect the time-varying volatility characteristics of financial asset return and the extreme-tail dependence characteristics of various financial assets return $[6,7]$. Meanwhile, Copula function can connect the joint distribution and the marginal distribution of multiple random variables to construct flexible multivariate distribution functions, which can be used to measure the extreme-tail dependence of multiple financial assets return. GARCH model can comprehensively describe the time-varying volatility characteristics of financial asset return. Therefore, building the multivariate time-varying $G$ $H$ Copula GARCH Model by combining the $G-H$ distribution with Copula function and GARCH model can not only comprehensively describe the "asymmetric, leptokurtic, and heavy-tail" characteristics, the time-varying volatility, and extreme-tail dependence characteristics of the financial asset return and make measurement of VaR more accurately, but also enrich and expand the risk measurement theory and method of financial market theoretically and improve the risk control ability of investors, corporations, financial institutions, and policy authorities and reduce their unnecessary losses in practice.

The $G-H$ distribution, Copula function, and GARCH model, as well as the financial risk measurement model based on them, have been researched in the existing relevant literatures. A lot of innovative research results with reference value have been brought out. Zhu and Pan [8] proposed three kinds of $G-H$ VaR methods based on the portfolio gains, losses, and extreme losses according to the statistical characteristics of $\mathrm{G}-\mathrm{H}$ distribution. Their empirical results showed that this method is superior to the commonly used delta-normal method. Kuester et al. [9] believed that the G$H$ distribution can describe skewness and kurtosis of the financial asset return simultaneously and it plays a very important role of VaR measurement of financial asset return. Degen et al. [10] discussed the application of $G-H$ distribution in operational risk measurement. Jondeau and Rockinger [11], Rodriguez [12], Fischer et al. [13], and Sun et al. [14] combined time series model with various Copulas functions by using the Sklar theorem to build a lot of highly flexible multivariate time-varying models for risk measurement of portfolios. Liu et al. [15] proposed a GARCH- $M$ model with a time-varying coefficient of the risk premium. Their study indicated that the coefficient of the risk premium varies with the time, and even in a mature market the conditional skewness in the return distribution is negatively correlated with the time-varying coefficient of the risk premium. Wen et al. [16] built a $D$ GARCH- $M$ model by separating investors' return into gains and losses on the basis of the characteristics of investors' risk preference. They found that investors become risk averse when they gain and risk-seeking when they lose, which effectively explains the inconsistent risk-return relationship. And the degrees of investors' risk aversion and risk-seeking are both in direct proportion to the value of gains and losses, respectively. Wen et al. [17] adopted aggregative indices of 14 representative stocks around the world as samples and established a TVRA-GARCH- $M$ model to investigate the influence of prior gains and losses on current risk attitude. The empirical results indicated that the prior gains increase people's current willingness to take risk asset at the whole market level. Huang et al. [18] combined Student's $t$-marginal distribution with Archimedean Copula functions to build the conditional Copula GARCH model. They used this model to estimate the VaR of portfolios. Ghorbel and Trabelsi [19] built the conditional extremum Copula GARCH model by using extreme value theory (EVT) and measured the risk of financial asset according to this model. Chollete et al. [20] used multivariate regime-switching Copula function to build international financial asset return model and accordingly put forward the VaR calculation method. Huggenberger and Klett [21] proposed a measurement model of multivariate risk asset return VaR based on $G-H$ distribution and Copula function. They used DAX30 (Germany), FTSE100 (UK), and CAC40 (French) from January 2000 to May 2010 as samples to test empirically. Wang et al. [22] applied the Gumbel Copula function in multivariate Archimedean Copula functions family to construct the joint distribution function which can describe the actual distribution and the correlation of various financial asset returns. They also used the Monte Carlo simulation technology to analyze the portfolios $\mathrm{VaR}$ and its composition under different confidence levels. The result showed that using the multidimensional Gumbel Copula function to construct the risk measurement model of financial asset can make the assets chosen by investors more robust, and it can also help investors to diversify and control the overall risk of the portfolios. Dai and Wen [23] proposed a computationally tractable robust optimization method for minimizing the CVaR of a portfolio under a general affine data perturbation uncertainty set. And they presented some numerical experiments with real market data to illustrate the behavior of robust optimization model. Liu et al. [24] proposed a pricing model for convertible bonds based on the utility-indifference method and got access to the empirical results by use of Information Technology. Furthermore, using the proposed theoretical model, they presented an empirical pricing study of China's market. They found that the theoretical prices are higher than the actual market prices $0.24-4.58 \%$ and the utilityindifference prices are better than the Black-Scholes (B-S) prices.

Based on the aforementioned analyses, the VaR is still the mainstream measurement method of financial market risk. In order to achieve the purpose of measuring $\mathrm{VaR}$ more precisely, it has been the hot issue of existing research literatures to construct the distribution functions as comprehensive as possible to describe the "asymmetric, leptokurtic, and heavy-tail" characteristics, the time-varying volatility characteristics, and the extreme-tail dependence characteristics of financial asset return through a variety of mathematical methods. However, in the process of constructing the return distribution model and measuring VaR of financial asset, existing results only grasp some characteristics of financial asset return distribution. They are not able to comprehensively describe the "asymmetric, leptokurtic, and heavy-tail" characteristics, the time-varying volatility characteristics, and the extreme-tail dependence characteristics of the financial asset return. The rationality and accuracy of VaR calculated based on the existing distribution models have a large space for further improvement. 
In this paper, we would take full advantage of the strengths of $G-H$ distribution, Copula function, and GARCH model in depicting the return distribution of financial asset to build multivariate time-varying $G-H$ Copula GARCH model which can simultaneously describe "asymmetric, leptokurtic, and heavy-tail" characteristics, the time-varying volatility characteristics, and the extreme-tail dependence characteristics of financial asset return and propose the estimation method of model parameters and the calculation algorithm of VaR. Then, this paper selects the SSCI (China), HSI (Hong Kong, China), TAIEX (Taiwan, China), and SP500 (USA) from January 3, 2000, to June 18, 2010, as samples to estimate the parameters and calculate the VaRs of various index portfolios under different confidence levels.

\section{2. $G-H$ Distribution, Copula Function, and Its Tail Dependence Index}

\subsection{G-H Distribution}

2.1.1. G Distribution. Assuming that random variable $Z$ obeys the standard normal distribution and $g$ is a real number, then the random variable $Y_{g}=G_{g}(z)$ obeys $G$ distribution. Consider

$$
G_{g}(z)=\frac{e^{g z}-1}{g}, \quad z \sim N(0,1),
$$

where $g$ controls the skewness of $G$ distribution. When $g \rightarrow$ $0, G_{g}(z) \rightarrow z$ and $G$ distribution tends to be symmetric. With the increase of the absolute value of $g$, the degree of asymmetry increases. Changing the sign of $g$ can change the asymmetric direction of $G$ distribution, but it does not change its degree of asymmetry.

2.1.2. $H$ Distribution. Assuming that random variable $Z$ obeys the standard normal distribution and $h$ is a real number, then the random variable $Y_{h}=H_{h}(z)$ obeys $H$ distribution. Consider

$$
H_{h}(z)=e^{h z^{2} / 2}, \quad z \sim N(0,1) .
$$

$H$ distribution stretches the tail of the standard normal distribution. $h$ controls the tail heaviness of $H$ distribution. The larger the $h$ is, the heavier the tail is. Because $H_{h}(z)$ is an even function, $H$ distribution is symmetric. But the heaviness of its tail changes compared to the standard normal distribution.

2.1.3. G-H Distribution. The random variable $Y_{g, h}$ can be obtained by introducing both functions $G_{g}(z)$ and $H_{h}(z)$ to revise standard normal random variable $Z$. Consider

$$
Y_{g, h}=G_{g}(z) H_{h}(z)=\frac{e^{g z}-1}{g} e^{h z^{2} / 2} .
$$

Then, $X_{g, h}$ can be obtained through linear transformation of $Y_{g, h}$. Consider

$$
X_{g, h}=A+B \frac{e^{g z}-1}{g} e^{h z^{2} / 2}, \quad z \sim N(0,1) .
$$

The distribution of the random variable $X_{g, h}$ obeys the $G$ $H$ distribution. $A, B, g$, and $h$ are real numbers. $g$ describes the asymmetry of $G-H$ distribution, and $h$ describes the heavy-tail characteristics of $G-H$ distribution. Obviously, (3) is a special form of (4). The random variable $Y_{g, h}$ in (3) is the random variable of $G-H$ distribution after central standardization.

2.2. Copula Function and Its Tail Dependence Index. Assuming that marginal distribution of random vector $u_{i}=F_{i}\left(x_{i}\right)$ $(i=1,2, \ldots, p)$ obeys uniform distribution $U(0,1)$, according to the Sklar theorem, the joint distribution function of $P$ dimensional random vectors $F\left(x_{1}, \ldots, x_{p}\right)$ can be represented as the following formula:

$$
F\left(x_{1}, \ldots, x_{p}\right)=C\left(F_{1}\left(x_{1}\right), \ldots, F_{p}\left(x_{p}\right)\right),
$$

where $C$ is the Copula function of $F$, which is a hypercube $[0,1]^{p}$ multivariate density function defined on $P$ dimensional space $\mathbf{R}^{p}$. If the marginal distribution is continuous, there is a unique Copula function $C$. Then

$$
C\left(u_{1}, \ldots, u_{p}\right)=F\left(F_{1}^{-1}\left(u_{1}\right), \ldots, F_{p}^{-1}\left(u_{p}\right)\right) .
$$

On the contrary, given $P$-dimensional Copula function $C\left(u_{1}, \ldots, u_{p}\right)$ and its marginal distribution function $F_{1}\left(x_{1}\right), \ldots, F_{p}\left(x_{p}\right)$, the density function of $P$-dimensional joint distribution function is

$$
f\left(x_{1}, \ldots, x_{p}\right)=c\left(F_{1}\left(u_{1}\right), \ldots, F_{p}\left(u_{p}\right)\right) \prod_{i=1}^{p} f_{i}\left(x_{i}\right) .
$$

If $f_{i}\left(x_{i}\right)$ is the edge density, $c\left(u_{1}, \ldots, u_{p}\right)$ denotes Copula density derived from (6). Thus,

$$
c\left(u_{1}, \ldots, u_{p}\right)=\frac{f\left(F_{1}^{-1}\left(u_{1}\right), \ldots, F_{p}^{-1}\left(u_{p}\right)\right)}{\prod_{i=1}^{p} f_{i}\left(F_{i}^{-1}\left(u_{i}\right)\right)} .
$$

Since the joint distribution function of random variables defines the correlation among its components, Copula function determines the dependent structure among random variables uniquely. The upper tail index $\lambda_{u}$ and lower tail index $\lambda_{l}$ of tail dependence indicators can be defined as follows:

$$
\begin{aligned}
& \lambda_{u}=\lim _{q \rightarrow 1} \frac{1-2 q+c(q, q)}{1-q}, \\
& \lambda_{l}=\lim _{q \rightarrow 0} \frac{c(q, q)}{q} .
\end{aligned}
$$

According to Nelsen [25], Gauss Copula function generated by multivariate normal distribution function whose correlation matrix is $\mathbf{R}$ can be represented as follows:

$$
\begin{aligned}
& C_{\mathbf{R}}^{G u}\left(u_{1}, \ldots, u_{p}\right) \\
& =\int_{-\infty}^{\Phi_{1}^{-1}\left(u_{1}\right)} \cdots \int_{-\infty}^{\Phi_{p}^{-1}\left(u_{p}\right)} \frac{1}{\sqrt{(2 \pi)^{p}|\mathbf{R}|}} \exp \left\{\frac{-\mathbf{u}^{\prime} \mathbf{R}^{-1} \mathbf{u}}{2}\right\} d \mathbf{u},
\end{aligned}
$$


where $\mathbf{u}=\left(u_{1}, \ldots, u_{p}\right)$ and $\Phi^{-1}$ is the inverse function of single normal distribution. Because the Gauss Copula function does not have the characteristics of tail dependence, we often use the $T$-Copula function whose degree of freedom is $\eta$ and correlation matrix is $\mathbf{R}$ to measure tail dependence structure of risk asset in empirical analysis; that is,

$$
\begin{aligned}
& C_{\eta, \mathbf{R}}^{t}\left(u_{1}, \ldots, u_{p}\right) \\
& =\int_{-\infty}^{t_{\eta}^{-1}\left(u_{1}\right)} \cdots \int_{-\infty}^{t_{\eta}^{-1}\left(u_{p}\right)} \frac{\Gamma((\eta+p) / 2)\left(1+\mathbf{u}^{\prime} \mathbf{R}^{-1} \mathbf{u} / 2\right)^{-(\eta+p) / 2}}{\Gamma(\eta / 2) \sqrt{(\pi \eta)^{p}|\mathbf{R}|}} d \mathbf{u},
\end{aligned}
$$

where $t_{\eta}^{-1}$ is the inverse function of simple standard Student's $t$-distribution whose degree of freedom is $\eta$. When $\eta \rightarrow \infty$, $T$-Copula function degenerates to Gauss Copula function. Its tail index $\lambda_{u}=\lambda_{l}=0$; that is, the tail is independent. The tail index of $T$-Copula function is

$$
\lambda_{u}=\lambda_{l}=2 t_{\eta+1}\left(-\frac{\sqrt{(\eta+1)(1-\rho)}}{\sqrt{1+\rho}}\right) \text {, }
$$

where $t_{\eta+1}$ is simple standard Student's $t$-distribution whose degree of freedom is $\eta+1$. Considering that the innovation impacts on the price of risk asset in varying degrees at different times, $\eta$ and $\rho$ should have time-varying characteristics. For this reason, tail index also has the same characteristics.

\section{Multivariate Time-Varying $G-H$ Copula GARCH Model}

Let $\mathbf{r}_{t}=\left(r_{1, t}, \ldots, r_{p, t}\right)$ denote return time series of $p$ risk assets. The prior information set before time $t$ is

$$
\mathbf{I}_{t-1}=\left\{\mathbf{r}_{t-1}, \mathbf{h}_{t-1}, \mathbf{r}_{t-2}, \mathbf{h}_{t-2}, \ldots\right\}=\prod_{i=1}^{p} \mathbf{I}_{i, t-1},
$$

where $\mathbf{I}_{i, t-1}=\left\{r_{i, t-1}, h_{i, t-1}, r_{i, t-2}, h_{i, t-2}, \ldots\right\} . h_{i, t}$ is conditional volatility of $r_{i, t}$ about single asset prior information set $\mathbf{I}_{i, t-1}$. Let $C\left(\cdot \mid \mathbf{I}_{t-1}\right)$ denote $P$-dimensional conditional Copula function and $F_{i}\left(r_{i, t} \mid \mathbf{I}_{i, t-1}\right)$ be the conditional distribution of the $i$ th component. According to Sklar theorem, the conditional joint distribution of $p$ risk assets return is

$$
\begin{aligned}
& F\left(\mathbf{r}_{t} \mid \mathbf{I}_{t-1}\right) \\
& \quad=C\left(F_{1}\left(r_{1, t} \mid \mathbf{I}_{1, t-1}\right), \ldots, F_{p}\left(r_{p, t} \mid \mathbf{I}_{p, t-1}\right) \mid \mathbf{I}_{t-1}\right) .
\end{aligned}
$$

Numerous empirical studies show that the risk asset return series obey GARCH $(1,1)$ model. Based on this, assuming that $r_{i, t}$ satisfies the GARCH $(1,1)$ model, we can get the following $G-H$ Copula GARCH $(1,1)$ model which describes the time-varying dependence structure of $p$ risk assets return after filtering the time-varying characteristics of single series:

$$
\begin{aligned}
& r_{i, t}=\mu_{i}+\varepsilon_{i, t}, \quad i=1,2, \ldots, p, \\
& \varepsilon_{i, t}=\sqrt{h_{i, t}} z_{i, t}, \\
& h_{i, t}=\omega_{i}+\alpha_{i} \varepsilon_{i, t-1}^{2}+\beta_{i} h_{i, t-1}, \\
& F\left(\mathbf{z}_{t} \mid \mathbf{I}_{t-1}\right) \\
& \quad=C\left(F_{1}\left(z_{1, t} \mid \mathbf{I}_{1, t-1}\right), \ldots, F_{p}\left(z_{p, t} \mid \mathbf{I}_{p, t-1}\right) \mid \mathbf{I}_{t-1}\right),
\end{aligned}
$$

where the parameters satisfy the conditions $\omega_{i}, \alpha_{i}, \beta_{i}>0$ and $\alpha_{i}+\beta_{i}<1$. These parameters can ensure the stability of conditional volatility series. The innovation series $\left\{\mathbf{z}_{t}\right\}$ obey $G-H$ distribution whose parameter is $(g, h)$ in $(4)$. But in order to simplify the analysis, we only consider $G-H$ distribution after central standardization given by (3) and its density function is written as $f_{Y_{i}}\left(y_{i}\right)$. The Copula function $C\left(\cdot \quad \mid \quad \mathbf{I}_{t-1}\right)$ is given by (11) and its density $c(\cdot)$ can be represented as the following time-varying $T$-Copula function whose degree of freedom is $\eta$ :

$$
\begin{aligned}
c_{\eta, \rho_{t}}^{t}\left(u_{1, t}, \ldots, u_{p, t}\right) \\
=\frac{f_{\eta, \rho_{t}}^{t}\left(f_{v_{1}}^{-1}\left(u_{1, t}\right), \ldots, f_{v_{p}}^{-1}\left(u_{p, t}\right)\right)}{\prod_{i=1}^{p} f_{\eta}\left(f_{v_{i}}^{-1}\left(u_{i, t}\right)\right)},
\end{aligned}
$$

where $f_{\eta, \rho_{t}}^{t}$ denotes the multivariate Student's $t$-distribution whose degree of freedom is $\eta$ and time-varying correlation matrix is $\boldsymbol{\rho}_{t}=\left(\rho_{i, j, t}\right)_{p \times p}$, and

$$
\rho_{i, i, t}=1
$$

$$
f_{v_{i}}\left(u_{i, t}\right)=\frac{\Gamma\left(\left(v_{i}+1\right) / 2\right)}{\Gamma\left(v_{i} / 2\right) \sqrt{v_{i} \pi}}\left(1+\frac{u_{i, t}^{2}}{v_{i}}\right)^{-\left(v_{i}+1\right) / 2} .
$$

The joint density function of $p$ risk assets return is

$$
\begin{gathered}
f\left(\mathbf{y} \mid \mathbf{u}, \mathbf{h}_{t}\right)=c_{\eta, \boldsymbol{\rho}_{t}}^{t}\left(x_{1, t}, \ldots, x_{p, t}\right) \prod_{i=1}^{p} f_{Y_{i}}\left(y_{i}\right) \\
=\Gamma\left(\frac{\eta+p}{2}\right) \Gamma\left(\frac{\eta}{2}\right)^{p-1}\left(1+\frac{\mathbf{x}_{t}^{\prime} \boldsymbol{\rho}_{t} \mathbf{x}_{t}}{\eta}\right)^{-(\eta+p) / 2} \\
\cdot\left(\left|\boldsymbol{\rho}_{t}\right|\right)^{-1 / 2} \prod_{i=1}^{p}\left(1+\frac{x_{i, t}^{2}}{\eta}\right)^{(\eta+1) / 2} \prod_{i=1}^{p} f_{Y_{i}}\left(y_{i}\right),
\end{gathered}
$$


where $\mathbf{x}_{t}=\left(x_{1, t}, \ldots, x_{p, t}\right)$ and $x_{i, t}=t_{\eta}^{-1}\left(t_{v_{i}}\left(\varepsilon_{i, t}\right)\right)$. Then the likelihood function of overall samples is

$$
\begin{aligned}
& l(\boldsymbol{\theta} \mid \mathbf{y})=\prod_{t=1}^{T} \Gamma\left(\frac{\eta+p}{2}\right) \Gamma\left(\frac{\eta}{2}\right)^{p-1} \\
& \cdot\left(1+\frac{\mathbf{x}_{t}^{\prime} \boldsymbol{\rho}_{t} \mathbf{x}_{t}}{\eta}\right)^{-(\eta+p) / 2}\left(\left|\boldsymbol{\rho}_{t}\right|\right)^{-1 / 2} \Gamma\left(\frac{\eta+1}{2}\right)^{-p} \\
& \cdot \prod_{i=1}^{p}\left(1+\frac{x_{i, t}^{2}}{\eta}\right)^{(\eta+1) / 2} \prod_{i=1}^{p} f_{Y_{i}}\left(y_{i}\right),
\end{aligned}
$$

where $\boldsymbol{\theta}=\left\{\left(\mu_{i},{\omega_{i}}_{i}, \alpha_{i}, \beta_{i}, v_{i}\right)_{i=1}^{p}, a, b, \boldsymbol{\rho}_{t}, \eta\right\}$ and $\mathbf{x}_{t}=\left(x_{1, t}, \ldots\right.$, $\left.x_{p, t}\right)$. The value of correlation matrix $\boldsymbol{\rho}_{t}$ is similar to the timevarying correlation matrix of multivariate Copula GARCH model proposed by Jondeau and Rockinger [11]. That is, $\boldsymbol{\rho}_{t}$ satisfies the following evolution equation

$$
\boldsymbol{\rho}_{t}=(1-a-b) \boldsymbol{\rho}+a \Psi_{t-1}+b \boldsymbol{\rho}_{t-1}
$$

where $0 \leq a, b \leq 1, a+b \leq 1$. $\rho$ is a positive definite matrix whose main diagonal elements are 1 and other elements are static correlation coefficients. $\Psi_{t-1}$ is a $p \times p$ matrix, in which every element

$$
\psi_{i, j, t-1}=\frac{\sum_{l=1}^{m} x_{i, t-l} x_{j, t-l}}{\sqrt{\sum_{l=1}^{m} x_{i, t-l}^{2}} \sqrt{\sum_{l=1}^{m} x_{j, t-l}^{2}}}, \quad i, j=1,2, \ldots, p
$$

denotes the correlation coefficients of $p$ risk asset returns, $(m \geq p+2), x_{t}=\left(x_{1, t}, \ldots, x_{p, t}\right)=\left(t_{v_{1}}^{-1}\left(f_{v_{1}}\left(z_{1, t}\right)\right), \ldots\right.$, $\left.t_{v_{p}}^{-1}\left(f_{v_{p}}\left(z_{p, t}\right)\right)\right)$. Each element $\rho_{i, j, t}$ of $\boldsymbol{\rho}_{t}$ satisfies $-1 \leq \rho_{i, j, t} \leq 1$.

\section{Parameter Estimation Algorithm of the Multivariate Time-Varying $G-H$ Copula GARCH Model}

On the basis of Huggenberger and Klett [21], this section will use dynamic correlation matrix $\boldsymbol{\rho}_{t}$ instead of static correlation matrix in the multidimensional discrete-time stochastic process to estimate the parameters of multivariate timevarying $G-H$ Copula GARCH model established in Section 3. Assuming that $\Theta$ denotes the parameter space defined by the model and $\left(\mathbf{r}_{1}, \mathbf{r}_{2}, \ldots, \mathbf{r}_{T}\right)$ denotes the log return samples of $P$-dimensional risk asset which is generated by multivariate conditional density function $f_{\boldsymbol{\rho}_{t} \mid \mathfrak{I}_{t-1}}\left(\mathbf{r}_{t} \mid \mathfrak{\Im}_{t-1}, \boldsymbol{\theta}_{0}\right)$, where $\boldsymbol{\theta}_{0} \in$ $\boldsymbol{\Theta}, \mathfrak{\Im}_{t-1}$ is $\sigma$ algebra of time $t-1$ and before, the maximum likelihood estimation of parameter vector $\boldsymbol{\theta}$ can be calculated by the following equation:

$$
\widehat{\boldsymbol{\theta}}=\underset{\boldsymbol{\theta} \in \boldsymbol{\Theta}}{\arg \max } \sum_{t=1}^{T} \log f_{\boldsymbol{\rho}_{t} \mid \mathfrak{\Im}_{t-1}}\left(\mathbf{r}_{t} \mid \mathfrak{\Im}_{t-1}, \boldsymbol{\theta}\right),
$$

where $f_{\boldsymbol{\rho}_{t} \mid \Im_{t-1}}\left(\mathbf{r}_{t} \mid \mathfrak{\Im}_{t-1}, \boldsymbol{\theta}\right)$ can be obtained by calculating the derivative of (5). Let $c_{\boldsymbol{\theta}}$ denote Copula density function. Thus

$$
\begin{aligned}
f_{\boldsymbol{\rho}_{t} \mid \Im_{t-1}}\left(r_{1, t}, \ldots, r_{p, t} \mid \mathfrak{\Im}_{t-1}, \boldsymbol{\theta}\right) \\
=c_{\boldsymbol{\theta}}\left(F_{1, t}\left(r_{1, t}, \boldsymbol{\theta}\right), \ldots, F_{p, t}\left(r_{p, t}, \boldsymbol{\theta}\right)\right) \\
\cdot \prod_{i=1}^{p} f_{i, t}\left(r_{t, i}, \boldsymbol{\theta}\right) .
\end{aligned}
$$

The probability density function and distribution function can be obtained in the process of model built in Section 3. Using the IFM method proposed by Joe [26], we can convert (22) into an optimization problem. Therefore, we need to divide the parameter vector $\boldsymbol{\theta}$ into two subparameter vectors $\boldsymbol{\theta}_{c}$ and $\boldsymbol{\theta}_{r}$ : that is $\boldsymbol{\theta}=\left(\boldsymbol{\theta}_{c}, \boldsymbol{\theta}_{r}\right)$, where $\boldsymbol{\theta}_{r}=\left(\boldsymbol{\theta}_{r_{1}}, \ldots, \boldsymbol{\theta}_{r_{p}}\right)$, $\boldsymbol{\theta}_{r_{i}}$ is the parameter vector of $i$ th marginal distribution, and $\boldsymbol{\theta}_{c}$ is the parameter vector of Copula function. Because IFM method is a two-step likelihood estimation method, the model parameters should be estimated through the following two steps.

Step 1. Solving the maximum likelihood estimator of the parameter vector of each risk asset return,

$$
\widehat{\boldsymbol{\theta}}_{r_{i}}=\underset{\boldsymbol{\theta}_{r_{i}}}{\arg \max } \sum_{t=1}^{T} \log f_{i, t}\left(r_{i, t} \mid \boldsymbol{\theta}_{r_{i}}\right) \quad i=1,2, \ldots, p .
$$

This means that we need to estimate parameters vector $\widehat{\boldsymbol{\theta}}_{r_{i}}$ of $p$ distributions continuously.

Step 2. Taking each $\widehat{\boldsymbol{\theta}}_{r_{i}}$ into the likelihood equation (22), we can obtain the parameter vector $\boldsymbol{\theta}_{c}$ of Copula function and its maximum likelihood estimator $\widehat{\boldsymbol{\theta}}_{c}$. Consider

$$
\begin{aligned}
& \widehat{\boldsymbol{\theta}}_{c} \\
& =\underset{\boldsymbol{\theta}_{c}}{\arg \max } \sum_{t=1}^{T} \log c_{\boldsymbol{\theta}_{c}}\left(F_{1, t}\left(\left|r_{1, t}\right| \widehat{\boldsymbol{\theta}}_{r_{1}}\right), \ldots, F_{p, t}\left(\left|r_{p, t}\right| \widehat{\boldsymbol{\theta}}_{r_{p}}\right)\right) .
\end{aligned}
$$

In the maximum likelihood estimation, we need to use the derivative function of the density function of $G-H$ marginal distribution with respect to the component of parameter vector. Because the density function of $G-H$ marginal distribution is very complex, this paper uses the implicit function differentiation rule to take its derivative. The estimator $\widehat{\boldsymbol{\theta}}_{2 s}$ of parameter vector $\boldsymbol{\theta}$ obtained by the above-mentioned two-step method obeys normal distribution consistently and asymptotically under the standard regularity conditions proposed in Huggenberger and Klett [21], Joe [26], and Patton [27]; that is,

$$
\sqrt{T}\left(\widehat{\boldsymbol{\theta}}_{2 s, T}-\boldsymbol{\theta}_{0}\right) \underset{T \rightarrow \infty}{\stackrel{d}{\longrightarrow}} N\left(0, \boldsymbol{\Omega}^{-1} \boldsymbol{\Sigma} \boldsymbol{\Omega}\right),
$$


where

$$
\begin{aligned}
\boldsymbol{\Omega} & =-E\left(\frac{\partial^{2}}{\partial \boldsymbol{\theta} \partial \boldsymbol{\theta}^{\prime}} \log f_{\boldsymbol{\rho}_{t} \mid \Im_{t-1}}\left(\boldsymbol{\rho}_{t} \mid \mathfrak{\Im}_{t-1}, \boldsymbol{\theta}_{0}\right)\right) \\
\boldsymbol{\Sigma} & =E\left(\frac{\partial}{\partial \boldsymbol{\theta}}\left(\log f_{\boldsymbol{\rho}_{t} \mid \Im_{t-1}}\left(\boldsymbol{\rho}_{t} \mid \mathfrak{\Im}_{t-1}, \boldsymbol{\theta}_{0}\right)\right)\right. \\
& \left.\cdot \frac{\partial}{\partial \boldsymbol{\theta}}\left(\log f_{\boldsymbol{\rho}_{t} \mid \Im_{t-1}}\left(\boldsymbol{\rho}_{t} \mid \mathfrak{\Im}_{t-1}, \boldsymbol{\theta}_{0}\right)\right)^{\prime}\right) .
\end{aligned}
$$

Because the matrixes $\boldsymbol{\Sigma}$ and $\boldsymbol{\Omega}$ can be estimated by the estimated parameter vector consistently,

$$
\begin{gathered}
\widehat{\boldsymbol{\Omega}}_{T}=-T^{-1} \sum_{t=1}^{T} \frac{\partial^{2}}{\partial \boldsymbol{\theta} \partial \boldsymbol{\theta}^{\prime}} \log f_{\boldsymbol{\rho}_{t} \mid \Im_{t-1}}\left(\mathbf{r}_{t} \mid \mathfrak{\Im}_{t-1}, \widehat{\boldsymbol{\theta}}_{2 s, T}\right), \\
\widehat{\boldsymbol{\Sigma}}_{T}=T^{-1} \sum_{t=1}^{T} \frac{\partial}{\partial \boldsymbol{\theta}}\left(\log f_{\boldsymbol{\rho}_{t} \mid \mathfrak{\Im}_{t-1}}\left(\mathbf{r}_{t} \mid \mathfrak{\Im}_{t-1}, \widehat{\boldsymbol{\theta}}_{2 s, T}\right)\right) \\
\cdot \frac{\partial}{\partial \boldsymbol{\theta}}\left(\log f_{\boldsymbol{\rho}_{t} \mid \mathfrak{\Im}_{t-1}}\left(\mathbf{r}_{t} \mid \mathfrak{\Im}_{t-1}, \widehat{\boldsymbol{\theta}}_{2 s, T}\right)\right)^{\prime} .
\end{gathered}
$$

Thus (26) can be used to calculate the standard deviation of the estimator $\widehat{\boldsymbol{\theta}}_{2 s, T}$.

\section{VaR Algorithm Based on the Multivariate Time-Varying $G-H$ Copula GARCH Model}

After estimating the parameters of multivariate time-varying $G-H$ Copula GARCH model, VaR of the risk portfolio can be measured. VaR of risk portfolio indicates the expected maximum losses of risk portfolio held by investors within a given confidence level and a certain period of time. Assuming that $\mathbf{r}_{t}=\left(r_{1, t}, \ldots, r_{p, t}\right)(t=1,2, \ldots, T)$ are the return samples of $p$ risk assets which satisfy the multivariate time-varying $G$ $H$ Copula GARCH $(1,1)$ model in Section 3 and $\sum_{i=1}^{p} \lambda_{i} r_{i, t}$ is the portfolio of $p$ risk assets in which the weight of the risk asset $i$ is $\lambda_{i}(i=1,2, \ldots, p)$ that can be less than 0 because of permitting short-purchasing and short-selling the risk assets and meet $\sum_{i=1}^{p} \lambda_{i}=1$, the $\mathrm{VaR}$ of risk portfolio under confidence level $q$ at time $t$ should satisfy $\operatorname{Pr}\left(\sum_{i=1}^{p} \lambda_{i} r_{i, t} \leq\right.$ $\left.\mathrm{VaR}_{t}\right)=q$. The confidence level $q$ can reflect the different risk preferences of investors or financial institutions to a certain extent. Choosing a larger confidence level means that investors or financial institutions have greater risk aversion, and they hope to get a forecast result with larger probability.

Although the conditional distributions of $r_{1, T+1}, r_{2, T+1}$, $\ldots, r_{p, T+1}$ can be calculated through the known marginal distributions, it is very difficult to calculate quantile from time-varying Copula density function, and it is adverse to measure and calculate the VaR of risk portfolio. Therefore, this paper measures the dynamic risk of portfolio and its estimation value approximately through simulating $G-H$ Copula GARCH model. Based on the parameters $\theta^{(n)}$ of the sample, the return series of risk assets $\left\{\left[r_{1,1+T}^{(n, m)}, \ldots, r_{p, 1+T}^{(n, m)}\right], m=\right.$ $1, \ldots, M\}$ and the one-step measurement and estimation values of $\mathrm{VaR}$ of their portfolios can be obtained through estimating $\left(h_{1, T+1}^{(n)}, \ldots, h_{p, T+1}^{(n+1)}\right)$ according to the volatility equation
TABLE 1: Moment estimation results of the daily log return of SSCI, HSI, TAIEX, and SP500.

\begin{tabular}{lcccc}
\hline Types of stock index & Mean & Std. & Skewness & Kurtosis \\
\hline SSCI & $2.5078 e-004$ & 0.0181 & -0.2144 & 7.4467 \\
HSI & $7.4505 e-005$ & 0.0177 & -0.2765 & 11.6866 \\
TAIEX & $3.9511 e-005$ & 0.0140 & -0.9091 & 17.2823 \\
SP500 & $-9.7173 e-005$ & 0.0148 & -0.3701 & 12.0177 \\
\hline
\end{tabular}

of $G-H$ Copula GARCH model and calculating $\rho_{T+1}^{(n)}$ by using Copula dynamic evolution equation and then repeating the following algorithm for $M$ times $(M \geq 3 p)$.

Step 1. It is simulating $M$ groups of random vectors $\left[u_{1, T+1}^{(n, m)}, \ldots, u_{p, T+1}^{(n, m)}\right]$ according to the multivariate $T$-Copula density function whose degree of freedom is $\eta^{(n)}$ and correlation matrix is $\rho_{T+1}^{(n)}$.

Step 2. Calculating $r_{i, T+1}^{(n, m)}=\mu_{i}^{(n)}+z_{i, T+1}^{(n, m)} \sqrt{h_{i, T+1}^{(n)}}, i=1, \ldots, p$.

Step 3. Firstly, one calculates the return rate of risk portfolio that is equal to $\sum_{i=1}^{p} \lambda_{i} r_{i, T+1}^{(n, m)}, m=1,2, \ldots, M$. Secondly, one evaluates its $q$-quantile $\operatorname{VaR}_{T+1}^{(n)}$. Thirdly, one measures the $\mathrm{VaR}$ of the risk portfolio by $\operatorname{VaR}_{T+1}=(1 / N) \sum_{n=1}^{N} \operatorname{VaR}_{T+1}^{(n)}$.

\section{Application of the Multivariate Time- Varying $G-H$ Copula GARCH Model}

6.1. Date Sample and Moment Estimation. USA and China, as the most developed capitalism country and the largest developing country in the world, respectively, rank top two of the world economy. Their stock markets should have strong representation in the world. At the same time, due to the historical reasons, there exist several regions with different political systems such as Mainland China, Hong Kong, Taiwan, and Macau in Greater China. Macau is similar to Hong Kong on the whole. For the above-mentioned reasons, this paper selects the SSCI (China), HSI (Hong Kong, China), TAIEX (Taiwan, China), and SP500 (USA) from January 3,2000 , to June 18,2010 , as data samples to estimate the $\mathrm{VaR}$ of various index portfolios under different confidence levels by using the multivariate time-varying $G-H$ Copula GARCH model. The data comes from Yahoo Finance website: http://finance.yahoo.com/.

The moment estimation results of the daily log return of SSCI, HSI, TAIEX, and SP500 are shown in Table 1.

Table 1 shows that the skewness of daily log returns of SSCI, HSI, TAIEX, and SP500 is less than 0 and their kurtosis is much larger than that of standard normal distribution which is equal to 3 . These results demonstrate that the daily log returns of these indices have the right skew and leptokurtic characteristics. Therefore, it is appropriate to fit the daily log return of SSCI, HSI, TAIEX, and SP500 by applying $G-H$ distribution which has leptokurtic, heavy-tail characteristics, and it is reasonable to apply the multivariate 
TABLE 2: Parameter estimates of the four-variate time-varying G-H Copula GARCH $(1,1)$ model based on SSCI index risk asset.

\begin{tabular}{lcccc}
\hline Model parameters & $\mu_{1}$ & $\omega_{1}$ & $\alpha_{1}$ & $\beta_{1}$ \\
\hline Estimate & $0.00025^{* * *}$ & $0.0551^{* * *}$ & $0.0617^{* * *}$ & $0.8583^{* * *}$ \\
$T$-statistic & 4.8653 & 4.6851 & 6.5764 & 4.4009 \\
\hline
\end{tabular}

Note: ${ }^{* *}$ in the table denotes that the parameter is significant at $1 \%$ level.

TABLE 3: Parameter estimates of the four-variate time-varying G-H Copula GARCH $(1,1)$ model based on HSI index risk asset.

\begin{tabular}{lcccc}
\hline Model parameters & $\mu_{2}$ & $\omega_{2}$ & $\alpha_{2}$ & $\beta_{2}$ \\
\hline Estimate & $0.000075^{* * *}$ & $0.0342^{* * *}$ & $0.0586^{* * *}$ & $0.8987^{* * *}$ \\
$T$-statistic & 4.6539 & 4.8518 & 6.1796 & 6.6079 \\
\hline
\end{tabular}

Note: ${ }^{* * *}$ in the table denotes that the parameter is significant at $1 \%$ level.

TABLE 4: Parameter estimates of the four-variate time-varying G-H Copula GARCH $(1,1)$ model based on TAIEX index risk asset.

\begin{tabular}{lcccc}
\hline Model parameters & $\mu_{3}$ & $\omega_{3}$ & $\alpha_{3}$ & $\beta_{3}$ \\
\hline Estimate & $0.00040^{* * *}$ & $0.0343^{* * *}$ & $0.0517^{* * *}$ & $0.9054^{* * *}$ \\
$T$-statistic & -6.4538 & 4.3523 & 5.9817 & 6.5935 \\
\hline
\end{tabular}

Note: ${ }^{* * *}$ in the table denotes that the parameter is significant at $1 \%$ level.

TABle 5: Parameter estimates of the four-variate time-varying G-H Copula GARCH $(1,1)$ model based on SP500 index risk asset.

\begin{tabular}{lcccc}
\hline Model parameters & $\mu_{4}$ & $\omega_{4}$ & $\alpha_{4}$ & $\beta_{4}$ \\
\hline Estimate & $-0.0001^{* * *}$ & $0.0251^{* * *}$ & $0.0861^{* * *}$ & $0.8738^{* * *}$ \\
$T$-statistic & -3.3873 & 5.2684 & 7.6324 & 6.3786 \\
\hline
\end{tabular}

Note: ${ }^{* * *}$ in the table denotes that the parameter is significant at $1 \%$ level.

time-varying $G-H$ Copula GARCH model to measure their VaR.

6.2. Parameter Estimates of the Multivariate Time-Varying G-H Copula GARCH Model. Based on the parameter estimation algorithm proposed in Section 4, the parameters of the multivariate time-varying $\mathrm{G}-\mathrm{H}$ Copula GARCH model with SSCI, HSI, TAIEX, and SP500 can be estimated. The parameter estimation results are shown in Tables 2, 3, 4, 5, and 6.

From Tables 2 to 6 , the following results can be obtained:

(1) Consider $\alpha_{1}+\beta_{1}=0.92, \alpha_{2}+\beta_{2}=0.9573, \alpha_{3}+$ $\beta_{3}=0.9571$, and $\alpha_{4}+\beta_{4}=0.9599$. This shows that the volatility persistence of Shanghai stock market is the strongest, Taiwan and Hong Kong stock market rank second and third, and the volatility persistence of USA stock market is minimum. It indicates that the investors' expectation of risk compensation in the emerging markets represented by China's stock market is stronger than that in the mature markets represented by the USA's stock market and the price discovery efficiency of innovation in the emerging markets represented by China's stock market is lower than that in the mature markets represented by USA's stock market. In addition, the sum of the coefficients $\alpha$ and $\beta$ is very close to 1 , which indicates that the impact and shock of innovation on the index volatility of each stock market has a long memory.

(2) The degree of freedom $\eta=14.57$ and the correlation coefficients $\rho_{i j}$ of T-Copula in Table 6 show that there exists the strongest correlation between Hong Kong stock market and Taiwan stock market, and the correlation between Shanghai stock market and Hong Kong stock market is also relatively large. The above-mentioned facts indicate that the extreme events probably result in the phenomena that Hong Kong stock market and Taiwan stock market are up and down synchronously, and there exist comoving behaviors between Shanghai stock market and Hong Kong stock market.

(3) The time-varying coefficient $b=0.987$ indicates that the time-varying correlation coefficient of $G$ $H$ Copula GARCH model has a long memory; that is, the impact of historical values of each other's correlation coefficient among SSCI, HSI, TAIEX, and SP500 on the expected correlation is relatively large.

6.3. VaR Measurement Based on the Multivariate TimeVarying G-H Copula GARCH Model. Based on the multivariate time-varying $G-H$ Copula GARCH model with SSCI, HSI, TAIEX, and SP500 whose parameters have been estimated, the VaRs of various index portfolios under different confidence levels can be measured. The measurement results are shown in Table 7.

From Table 7, the following results can be obtained:

(1) The inequalities $\operatorname{VaR}(S S C I)<\operatorname{VaR}(H S I)<V a R$ $(S P 500)<\operatorname{VaR}($ TAIEX) can be satisfied for any confidence level. It shows that the risk of extreme losses in Shanghai stock market is higher than that in Hong Kong stock market, Taiwan stock market, and USA stock market. This measurement result is in line with the actual situation that the maturity of Shanghai stock market is far lower than that of Hong Kong stock market, Taiwan stock market, and USA stock market.

(2) For any confidence level, the extreme losses risk of the investors who equally allocate their total assets among SSCI, HSI, TAIEX, and SP500 is lower than that of the investors who put their total assets into one index asset. The extreme losses risk of the investors increases with the concentration of risk asset in the index portfolios. This measurement result is consistent with the risk diversification theory of portfolio.

\section{Conclusion}

Considering the "asymmetric, leptokurtic, and heavy-tail" characteristics, the time-varying volatility characteristics, and extreme-tail dependence characteristics of financial asset return, this paper combined the $G-H$ distribution, Copula function, and GARCH model to construct a multivariate time-varying $\mathrm{G}-\mathrm{H}$ Copula GARCH model which can comprehensively describe the "asymmetric, leptokurtic, 
TABLE 6: Estimates of correlation coefficients, time-varying parameters, and degree of freedom of four-variate time-varying G-H Copula GARCH $(1,1)$ model based on SSCI, HSI, TAIEX, and SP500.

\begin{tabular}{|c|c|c|c|c|c|c|c|c|c|}
\hline Model parameters & $\rho_{12}$ & $\rho_{13}$ & $\rho_{14}$ & $\rho_{23}$ & $\rho_{24}$ & $\rho_{34}$ & $a$ & $b$ & $\eta$ \\
\hline Estimate & $0.256^{* * *}$ & $0.139^{* * *}$ & $0.021^{* * *}$ & $0.528^{* * *}$ & $0.193^{* * *}$ & $0.128^{* *}$ & $0.008^{* * *}$ & $0.987^{* * *}$ & $14.57^{* * *}$ \\
\hline$T$-statistic & 13.51 & 11.02 & 3.431 & 5.763 & 9.564 & 2.529 & 5.179 & 3.561 & 6.871 \\
\hline
\end{tabular}

Note: ${ }^{* *}$ and ${ }^{* *}$ in the table denote that the parameter is significant at $1 \%$ and $5 \%$ level, respectively.

TABLE 7: VaR estimation results based on the four-variate time-varying G-H Copula GARCH model with SSCI, HSI, TAIEX, and SP500.

\begin{tabular}{|c|c|c|c|c|c|c|c|c|c|}
\hline Ratio of index portfolio & $1 \%$ & $5 \%$ & $10 \%$ & $15 \%$ & Ratio of index portfolio & $1 \%$ & $5 \%$ & $10 \%$ & $15 \%$ \\
\hline $0.25: 0.25: 0.25: 0.25$ & -0.0303 & -0.0165 & -0.0116 & -0.0086 & $0.25: 0.25: 0.25: 0.25$ & -0.0303 & -0.0165 & -0.0116 & -0.0086 \\
\hline $0.50: 0.00: 0.25: 0.25$ & -0.0329 & -0.0177 & -0.0128 & -0.0093 & $0.25: 0.50: 0.00: 0.25$ & -0.0361 & -0.0189 & -0.0132 & -0.0100 \\
\hline $0.75: 0.00: 0.00: 0.25$ & -0.0418 & -0.0226 & -0.0157 & -0.0116 & $0.25: 0.75: 0.00: 0.00$ & -0.0426 & -0.0236 & -0.0165 & -0.0123 \\
\hline $1.00: 0.00: 0.00: 0.00$ & -0.0536 & -0.0285 & -0.0199 & -0.0150 & $0.00: 1.00: 0.00: 0.00$ & -0.0491 & -0.0269 & -0.0190 & -0.0138 \\
\hline $0.25: 0.25: 0.25: 0.25$ & -0.0303 & -0.0165 & -0.0116 & -0.0086 & $0.25: 0.25: 0.25: 0.25$ & -0.0303 & -0.0165 & -0.0116 & -0.0086 \\
\hline $0.25: 0.25: 0.50: 0.00$ & -0.332 & -0.0176 & -0.0119 & -0.0093 & $0.00: 0.25: 0.50: 0.50$ & -0.0359 & -0.0181 & -0.0128 & -0.0094 \\
\hline $0.00: 0.25: 0.75: 0.00$ & -0.0403 & -0.0196 & -0.0127 & -0.0097 & $0.00: 0.00: 0.25: 0.75$ & -0.0402 & -0.0206 & -0.0146 & -0.0108 \\
\hline $0.00: 0.00: 1.00: 0.00$ & -0.0420 & -0.0211 & -0.0139 & -0.0101 & $0.00: 0.00: 0.00: 1.00$ & -0.0425 & -0.0230 & -0.0157 & -0.0119 \\
\hline
\end{tabular}

Note: the ratio of index portfolio is ranked by the sequence of SSCI : HSI : TAIEX: SP500 in Table 7.

and heavy-tail" characteristics, the time-varying volatility characteristics, and extreme-tail dependence characteristics of financial asset return. It proposed the parameter estimation algorithm of the multivariate time-varying $G-H$ Copula GARCH model by using condition maximum likelihood method and IFM two-step method. An algorithm was constructed to calculate VaR by using the quantile function and the simulation method based on $G-H$ Copula GARCH model. In addition, this paper selected the daily log return of SSCI (China), HSI (Hong Kong, China), TAIEX (Taiwan, China), and SP500 (USA) from January 3, 2000, to June 18,2010 , as samples to estimate the parameters of the multivariate time-varying $G-H$ Copula GARCH model, and it also estimated the VaR for various index risk asset portfolios under different confidence levels. The research results showed that the multivariate time-varying G-H Copula GARCH model constructed in this paper could reasonably estimate and measure the extreme losses of risk portfolios in financial market, and the measurement results were in line with the actual situation of stock market and the risk diversification theory of portfolio. The achievement of this paper provided a practical and effective method for measuring the extreme losses of financial market.

\section{Conflict of Interests}

The authors declare that they have no conflict of interests regarding the publication of this paper.

\section{Acknowledgments}

The authors would like to thank the referees for all helpful comments and suggestions. This work is supported by Natural Science Foundation Project of CQ CSTC (Grant no. CSTC, 2011BB2088) and Key Project of National Natural Science Foundation (Grant no. 71232004).

\section{References}

[1] S. J. Kon, "Models of stock returns-a comparison," The Journal of Finance, vol. 39, no. 1, pp. 147-165, 1984.

[2] J. B. Gray and D. W. French, "Empirical comparisons of distributional models for stock index returns," Journal of Business Finance \& Accounting, vol. 17, no. 3, pp. 451-459, 1990.

[3] E. Jondeau and M. Rockinger, "Testing for differences in the tails of stock-market returns," Journal of Empirical Finance, vol. 10, no. 5, pp. 559-581, 2003.

[4] C. Jiang, S. Li, and S. Liang, "Empirical investigation of distribution feature of return in china stock market," Application of Statistics and Management, vol. 26, no. 4, pp. 710-717, 2007.

[5] D. Dong and W. Jin, "A subjective model of the distribution of returns and empirical analysis," Chinese Journal of Management Science, vol. 15, no. 1, pp. 112-120, 2007.

[6] J. Martinez and B. Iglewicz, "Some properties of the tukey g and $\mathrm{h}$ family of distributions," Communications in Statistics-Theory and Methods, vol. 13, no. 3, pp. 353-369, 1984.

[7] T. C. Mills, "Modelling skewness and kurtosis in the London stock exchange FT-SE index return distributions," The Statistician, vol. 44, no. 3, pp. 323-332, 1995.

[8] H. Zhu and Z. Pan, "A study of portfolios VaR method based on g-h distribution," Chinese Journal of Management Science, vol. 13, no. 4, pp. 7-12, 2005.

[9] K. Kuester, S. Mittnik, and M. S. Paolella, "Value-at-risk prediction: a comparison of alternative strategies," Journal of Financial Econometrics, vol. 4, no. 1, pp. 53-89, 2006.

[10] M. Degen, P. Embrechts, and D. Lambrigger, "The quantitative modeling of operational risk: between g-and-h and EVT," Astin Bulletin, vol. 37, no. 2, pp. 265-291, 2007.

[11] E. Jondeau and M. Rockinger, "The Copula-GARCH model of conditional dependencies: an international stock market application," Journal of International Money and Finance, vol. 25, no. 5, pp. 827-853, 2006.

[12] J. C. Rodriguez, "Measuring financial contagion: a Copula approach," Journal of Empirical Finance, vol. 14, no. 3, pp. 401423, 2007. 
[13] M. Fischer, C. Kock, S. Schluter, and F. Weigert, "An empirical analysis of multivariate copula models," Quantitative Finance, vol. 9, no. 7, pp. 839-854, 2009.

[14] W. Sun, S. Rachev, F. J. Fabozzi, and S. S. Kalev, "A new approach to modeling co-movement of international equity markets: evidence of unconditional copula-based simulation of tail dependence," Empirical Economics, vol. 36, no. 1, pp. 201229, 2009.

[15] Z. Liu, T. Zhang, and F. Wen, “Time-varying risk attitude and conditional skewness," Abstract and Applied Analysis, vol. 2014, Article ID 174848, 11 pages, 2014.

[16] F. Wen, Z. He, Z. Dai, and X. Yang, "Characteristics of investors' risk preference for stock markets," Economic Computation and Economic Cybernetics Studies and Research, vol. 3, no. 48, pp. 235-254, 2014.

[17] F. Wen, X. Gong, Y. Chao, and X. Chen, "The effects of prior outcomes on risky choice: evidence from the stock market," Mathematical Problems in Engineering, vol. 2014, Article ID 272518, 8 pages, 2014.

[18] J.-J. Huang, K.-J. Lee, H. Liang, and W.-F. Lin, "Estimating value at risk of portfolio by conditional copula-GARCH method," Insurance: Mathematics and Economics, vol. 45, no. 3, pp. 315324, 2009.

[19] A. Ghorbel and A. Trabelsi, "Measure of financial risk using conditional extreme value copulas with EVT margins," Journal of Risk, vol. 11, no. 4, pp. 51-85, 2009.

[20] L. Chollete, A. Heinen, and A. Valdesogo, "Modeling international financial returns with a multivariate regime-switching copula," Journal of Financial Econometrics, vol. 7, no. 4, pp. 437480, 2009.

[21] M. Huggenberger and T. Klett, "A g-and-h copula approach to risk measurement in multivariate financial models," Working Paper, 2010, http://ssrn.com/abstract=1677431.

[22] J. Wang, W. Bao, and J. Hu, "Portfolios VaR analyses based on the multi-dimensional gumbel copula function," Journal of Applied Statistics and Management, vol. 29, no. 1, pp. 137-143, 2010.

[23] Z. Dai and F. Wen, "Robust CVaR-based portfolio optimization under a genal affine data perturbation uncertainty set," Journal of Computational Analysis and Applications, vol. 16, no. 1, pp. 93-103, 2014.

[24] J. Liu, M. Tao, C. Ma, and F. Wen, "Utility indifference pricing of convertible bonds," International Journal of Information Technology and Decision Making, vol. 13, no. 2, pp. 429-444, 2014.

[25] R. B. Nelsen, An Introduction to Copulas, Springer Science \& Business Media, New York, NY, USA, 2007.

[26] H. Joe, Multivariate Models and Dependence Concepts, vol. 73 of Monographs on Statistics and Applied Probability, Chapman \& Hall, London, UK, 1997.

[27] A. J. Patton, "Estimation of multivariate models for time series of possibly different lengths," Journal of Applied Econometrics, vol. 21, no. 2, pp. 147-173, 2006. 


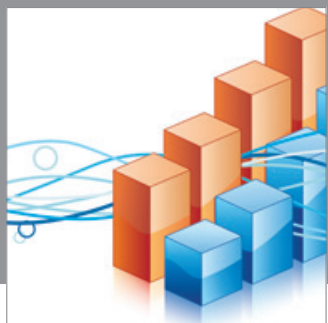

Advances in

Operations Research

mansans

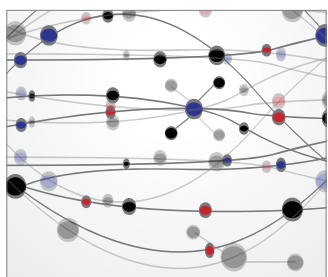

The Scientific World Journal
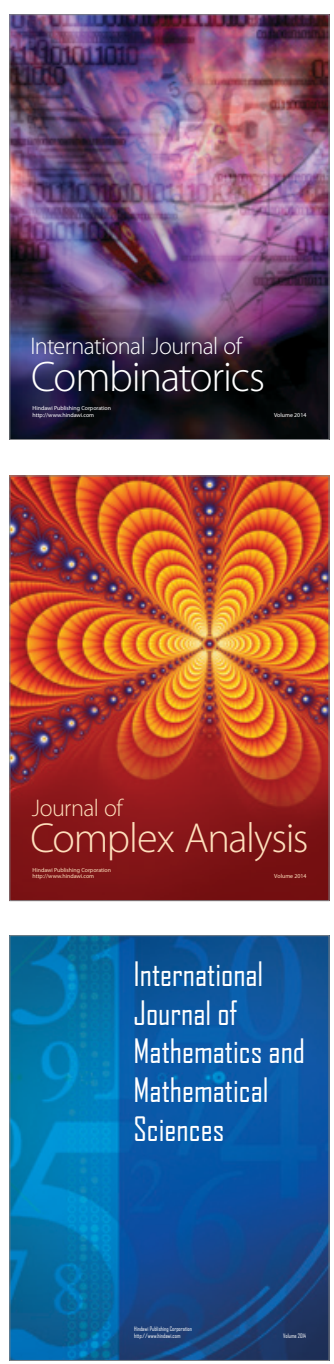
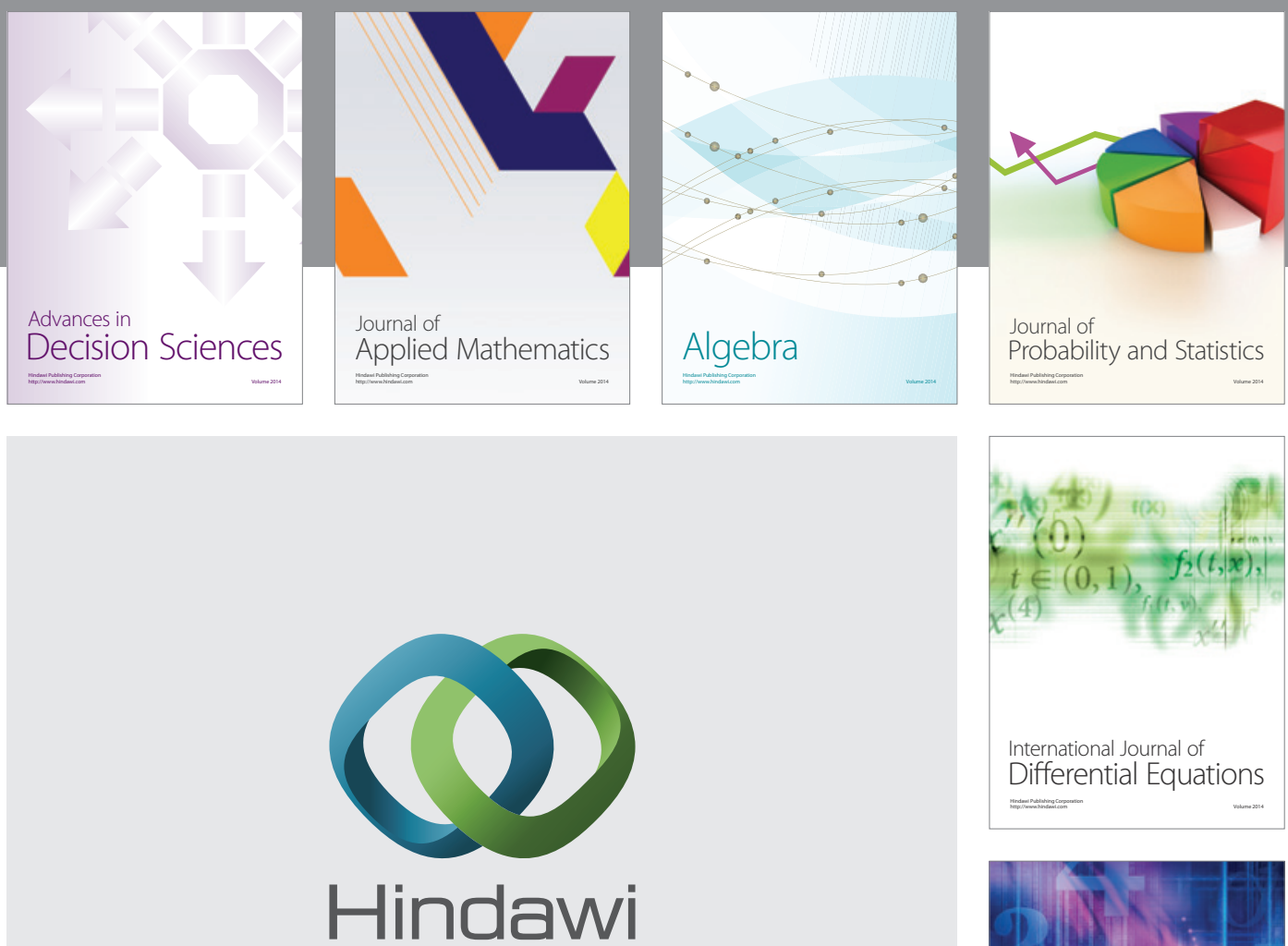

Submit your manuscripts at http://www.hindawi.com
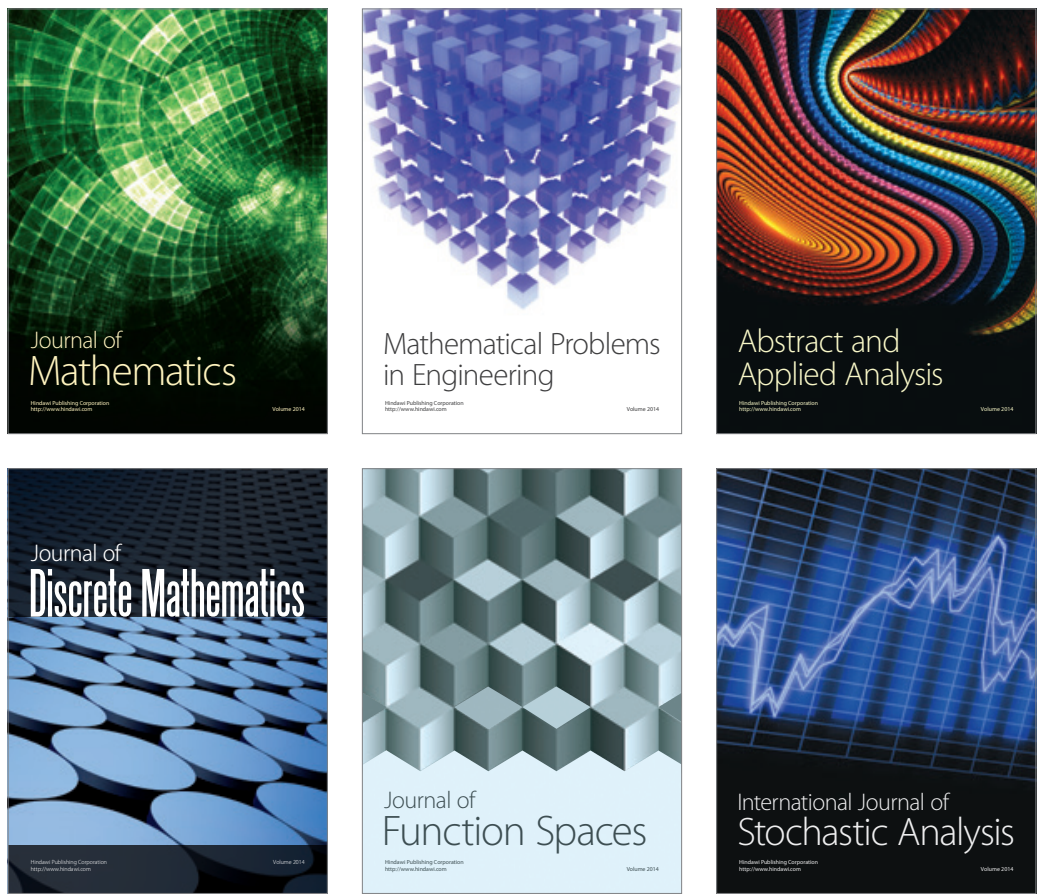

Journal of

Function Spaces

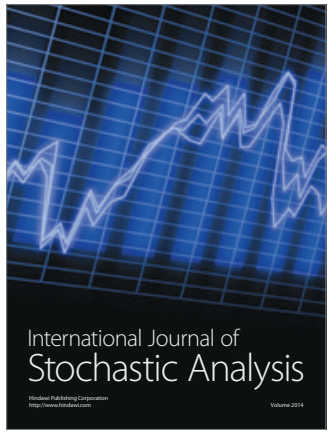

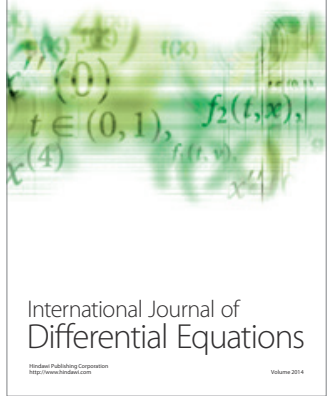
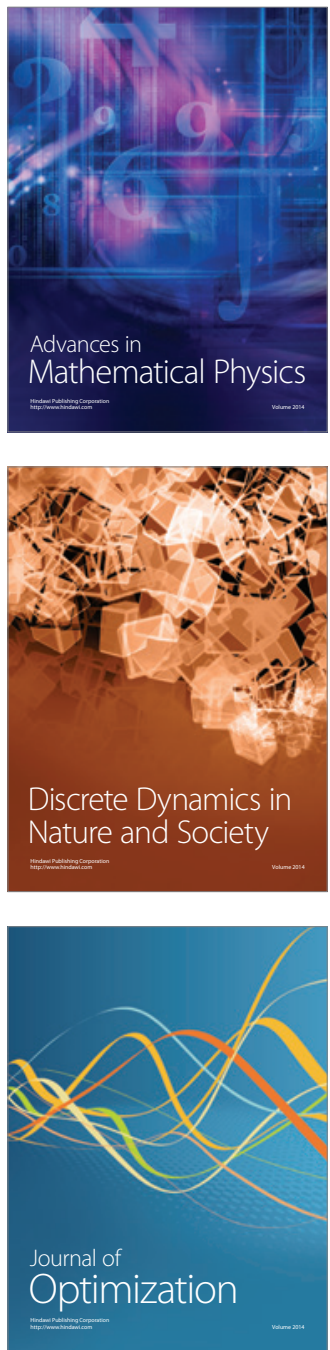